\title{
Improving Nutritional Status in a Pediatric Cystic Fibrosis Center
}

\author{
Ixsy Ramírez, MD, MPH, ${ }^{1}$ Amy Filbrun, MD, ms, ${ }^{1}$ Aws Hasan, Bs, $^{2}$ Kelley M. Kidwell, PhD, ${ }^{3}$ and \\ Samya Z. Nasr, MD, $\mathrm{CPI}^{1 *}$
}

\begin{abstract}
Summary. Background: The nutritional status of patients with cystic fibrosis (CF) is strongly associated with pulmonary function, respiratory status and survival. Malnutrition could result from a discrepancy between energy needs and food intake while malabsorption results from pancreatic insufficiency which occurs in $85 \%$ of people with CF. Methods: A quality improvement (QI) project was designed to improve the nutritional status of patients with CF with low Body Mass Index (BMI) between 3 and 19 years of age. An algorithm was developed which included clinic-based assessments of patients' nutritional status and periodic assessment by a dietitian, social worker and/or psychologist during the project. Gastrostomy tube placement and feeding was offered as a last resort to improve caloric intake. Results: 173 patients seen during January-June, 2010, were included in this project. They were classified into four BMl groups and data were collected quarterly through June, 2012. The project target population (BMI percentile $\leq 24$ ) had a median BMI percentile at the start of the project of 11.8. At the end of the project median BMI percentile was 22 (46\% improvement). Conclusion: Improving nutrition and BMI for patients with CF is achievable. There must be a motivated, multi-disciplinary team that includes patients and families. A patient-specific combination of interventions must be used. These interventions could be quite basic for patients with BMI percentile $\geq 25$, yet more elaborate for patients with BMI percentile $<25$. Clinic-based algorithms such as ours can successfully improve the BMI percentile in patients with CF. Pediatr Pulmonol. 2015;50:544-551. ๑ 2014 Wiley Periodicals, Inc.
\end{abstract}

Key words: nutrition; body mass index (BMI); pediatric; cystic Fibrosis; quality improvement; team approach.

Funding source: none reported.

\section{BACKGROUND}

Cystic Fibrosis (CF) is an autosomal recessive disease. It is the most common life shortening disease in Caucasians and also affects other ethnic groups. ${ }^{1}$ Median life expectancy for patients with CF is 36.8 years of age in 2012. ${ }^{1}$ CF affects mainly the respiratory tract and digestive system and to a lesser extent other systems in the body. ${ }^{1}$ Lung disease is a major cause of morbidity and mortality in patients with CF. Pulmonary function is the primary predictor of death. ${ }^{2}$ The rate of pulmonary function deterioration is the most important predictor of deterioration. ${ }^{3}$ Nutritional status of patients with $\mathrm{CF}$ is strongly associated with pulmonary function, respiratory status, and survival. ${ }^{4}$ One of the ways to improve pulmonary function and survival for patients with $\mathrm{CF}$ is to improve their nutritional status. It has therefore been recommended by the $\mathrm{CF}$ foundation (CFF) to maintain a $\mathrm{BMI} \geq 50$ th percentile for age given its association with improved lung function. ${ }^{5}$

Despite extensive efforts to improve CF patient nutritional status, less than half of the pediatric patients with $\mathrm{CF}$ in the United States have a BMI $\geq 50$ th percentile for age. ${ }^{1,5}$ Malnutrition most likely results from a discrepancy between energy needs and micronutrient requirements and food intake. In addition, there is malabsorption that results from pancreatic insufficiency

\footnotetext{
${ }^{1}$ Department of Pediatrics, Division of Pediatric Pulmonology University of Michigan Hospital and Health System, Ann Arbor, Michigan.

${ }^{2}$ Wayne State University Medical School, Detroit, Michigan.

${ }^{3}$ Department of Biostatistics, University of Michigan, Michigan.
}

No financial disclosures for any authors relevant to this project

Conflict of Interest: none

${ }^{*}$ Correspondence to: Samya Z. Nasr, MD, Professor of Pediatrics, 1500 E. Medical Center Dr Ann Arbor, MI. 48109-5212. E-mail: snasr@umich.edu Received 11 February 2014; Revised 4 June 2014; Accepted 3 July 2014

DOI 10.1002/ppul.23128

Published online 11 November 2014 in Wiley Online Library

(wileyonlinelibrary.com). 
which affects about $85 \%$ of patients with $\mathrm{CF}^{5-7}$ Also, patients with $\mathrm{CF}$ are at increased risk of other conditions that may affect their nutritional status. ${ }^{8} \mathrm{CF}$ related diabetes (CFRD) is present in approximately $16 \%$ of the $\mathrm{CF}$ population and has been linked to a decline in nutritional status and lung function. ${ }^{9}$

The CFF recommends that intensive treatment with both behavioral intervention and nutrition counseling be used to promote weight gain in children 1-12 years of age with growth deficits. ${ }^{5}$ For children with growth deficits and adults with weight deficits, the CFF recommends the use of nutritional supplements. ${ }^{5}$ Several studies were conducted to evaluate behavioral intervention and nutrition counseling in patients with CF 12 years of age and younger. ${ }^{4,10,11}$ These studies demonstrated significant improvement in patients' behavior around meal time and improvement in weight gain. On the other hand, one study was conducted in adult patients with $\mathrm{CF}$ to assess a 10week home based behavioral nutrition intervention. ${ }^{12}$ The study showed substantial improvements in the intervention group's specific CF nutrition knowledge and fat intake, but no substantial change in BMI or health related quality of life over time.

This is a prospective interventional project and was done as part of quality improvement efforts at the University of Michigan Pediatric Cystic Fibrosis Center (CFC). The project was conducted to improve the overall nutritional status of all pediatric patients with $\mathrm{CF}$ in the center in an effort to improve BMI to 50th percentile and above.

\section{METHODS}

\section{Setting}

This project was performed at the University of Michigan C.S. Mott Children's Hospital Cystic Fibrosis Center (CFC). This is a relatively large CFC that follows approximately 280 pediatric patients with $\mathrm{CF}$ and 480 individuals with $\mathrm{CF}$ in total. Adult patients are followed by adult pulmonologists at this center.

\begin{tabular}{|ll|}
\hline ABBREVIATIONS: \\
BMI & Body mass index \\
CF & Cystic fibrosis \\
CFC & Cystic fibrosis center \\
CFF & Cystic fibrosis foundation \\
CFRD & Cystic fibrosis related diabetes \\
FAB & Family advisory board \\
FEV $_{1 \%}$ & forced expiratory volume at 1 second, percent predicted \\
FEF $_{25-75 \%}$ & forced expiratory flow between 25 and 75 percent of \\
& exhaled volume, percent predicted \\
PDSA & Plan-do-study-act \\
QI & Quality improvement \\
SD & Standard deviation \\
& \\
\hline
\end{tabular}

\section{Study Population}

173 children and adolescents with a confirmed diagnosis of CF, age 3-19 years of age attending our center were included in the project. A CF diagnosis was confirmed through positive sweat chloride testing of $\geq 60 \mathrm{meq} / \mathrm{L}$ and/or $2 \mathrm{CF}$ disease causing mutations. Median sweat chloride testing was $100.5 \mathrm{mmol} / \mathrm{L}$ [Table 1]. Given that the focus was on BMI percentile, children ages 3 years of age and older were included. Patients were divided into four groups according to their BMI percentile. A color-coding system was utilized to quickly distinguish each BMI percentile category for easier identification by staff, patients and families. The BMI categories were: $\geq 50$ percentile [blue], 25-49 percentiles [yellow], 1024 percentiles [orange], and $<10$ percentile [red]. Patients in the lowest two groups [BMI $\leq 24$ percentile] were the specific target for the project. Project duration was 2.5 years.

\section{Intervention: Clinical Pathway Development}

A nutritional algorithm was developed to improve weight gain and BMI for all patients, with special focus on the targeted population [BMI $\leq 24$ percentile]. Developing the algorithm helped in standardization of care between team members. We also involved members of our CF Family Advisory Board (FAB) to review the algorithm so that they could provide the family perspective on the strengths and pitfalls of the algorithm before implementing the final version (Fig. 1).

The algorithm included assessment of patients' nutritional status at their regularly scheduled quarterly visits by the CFC registered dietitian. A high calorie diet is recommended for all patients with $\mathrm{CF}$ especially with a $\mathrm{BMI} \leq 50$ percentile. Estimated energy needs were in accordance with CF dietary guidelines of $120-150 \%$ of the recommended dietary allowance (RDA) per day. ${ }^{14}$ Oral supplements were recommended for the targeted patients [BMI $\leq 24$ percentile]. The recommended amount of oral supplements per day was determined by the caloric deficit to reach the patient's daily energy needs. Type of supplement and caloric density per ounce was determined by our CFC dietitian. Detailed assessment by a social worker and/or psychologist was done at the start of the project and periodically to help identify any obstacles to improved caloric intake. Appetite stimulants were recommended to patients with poor appetite. These included cyproheptadine as a first line treatment. Mirtazapine was offered if there was a concurrent diagnosis of depression and if it was clinically recommended by the patient's psychiatrist. Patients that were not able to gain weight with the above measures were recommended to be evaluated for gastrostomy tube placement to help improve caloric intake through nocturnal feeding. 


\begin{tabular}{|c|c|c|c|c|c|c|}
\hline Patient characteristic* & $\begin{array}{c}\text { Total } \\
\mathrm{N}=173\end{array}$ & $\begin{array}{c}\text { Group } 4 \\
\text { (BMI } \geq 50 \% \text { ile) } \\
\mathrm{N}=99\end{array}$ & $\begin{array}{c}\text { Group } 3 \\
(\text { BMI }=25-49 \% \text { ile) } \\
\mathrm{N}=37\end{array}$ & $\begin{array}{c}\text { Group } 2 \\
(\mathrm{BMI}=10-24 \% \text { ile }) \\
\mathrm{N}=20\end{array}$ & $\mathrm{~N}=17$ & $P$-value \\
\hline Age at CF Diagnosis, years (median) & 0.49 & 0.55 & 0.46 & 0.27 & 0.30 & 0.63 \\
\hline Age, years (median) & 11.43 & 10.95 & 11.27 & 12.79 & 13.10 & 0.41 \\
\hline Age, years (\%) & & & & & & 0.52 \\
\hline $2-6$ & 21.39 & 24.24 & 24.32 & 15.00 & 5.88 & \\
\hline $7-12$ & 40.46 & 41.41 & 40.54 & 35.00 & 41.18 & \\
\hline $13-19$ & 38.15 & 34.34 & 35.14 & 50.00 & 52.94 & \\
\hline Height, percentile (median) & 30.50 & 31.00 & 33.00 & 29.50 & 23.00 & 0.51 \\
\hline Weight, percentile (median) & 46.00 & 64.00 & 38.50 & 19.50 & 6.0 & $<0.001$ \\
\hline FEV (median) & 92.50 & 96.00 & 93.00 & 79.00 & 76.00 & $<0.001$ \\
\hline FEF 25-75 (median) & 83.50 & 89.00 & 84.00 & 65.00 & 42.00 & $<0.001$ \\
\hline Pseudomonas (\%) & 23.26 & 23.23 & 24.32 & 15.00 & 31.25 & 0.70 \\
\hline Sweat test, mmol/L (median) & 100.50 & 100.00 & 101.50 & 104.00 & 101.00 & 0.99 \\
\hline Gender $(\%)$ & & & & & & 0.81 \\
\hline Male & 49.13 & 48.48 & 56.76 & 55.00 & 47.06 & \\
\hline Female & 50.87 & 51.52 & 43.24 & 45.00 & 52.92 & \\
\hline G-tube $(\%)$ & 17.34 & 14.14 & 18.92 & 10.00 & 41.18 & 0.003 \\
\hline Appetite stimulant (\%) & 16.76 & 9.09 & 18.92 & 35.00 & 35.29 & 0.06 \\
\hline Pancreatic status (\%) & & & & & & 0.92 \\
\hline Insufficient & 83.77 & 82.76 & 87.50 & 80.00 & 86.67 & \\
\hline Sufficient & 16.23 & 17.24 & 12.50 & 20.00 & 13.33 & \\
\hline Genetic Category (\%) & & & & & & 0.012 \\
\hline Homozygous dF508 & 50.30 & 42.39 & 75.68 & 50.00 & 37.50 & \\
\hline Heterozygous dF508 & 36.97 & 45.65 & 13.51 & 35.00 & 43.75 & \\
\hline Other & 12.73 & 11.96 & 10.81 & 15.00 & 18.75 & \\
\hline
\end{tabular}

*Missing Data: 2 age of diagnosis, 3 from height and weight percentile, 19 from FEF and FEV, 1 from pseudomonas, 37 from sweat test, 19 from pancreatic status, 8 from genetic category.

All patients were given a weight gain goal per month at each clinical visit. This ranged from 0.25 to 1.5 pounds per month depending on the patient's age and weight. The team members worked with patients and families to address any obstacles in obtaining supplements, medications or insurance coverage concerns to facilitate access. Most of our intervention was clinic based. The targeted patient population was monitored more frequently than the rest of the CF population for weight gain, BMI and caloric intake when it was felt to be indicated. This included monthly rather than quarterly visits or phone calls to the family for updates on diet history and appetite. Our pediatric psychologist and social worker, in addition to the rest of the team, were available and offered follow up between clinic visits to help troubleshoot with patients and their families who were in greater need of assistance to provide more ongoing support if necessary.

At each clinic visit, children ages 6 and older underwent spirometry testing. Their $\mathrm{FEV}_{1} \%$ was followed at each clinic visit and compared to previous values to determine if these values were stable, improved or worsened. Only results which met American Thoracic Society criteria for interpretation were included in the study for analysis. Children less than 6 years of age did not undergo spirometry testing.
Several Plan-Do-Study-Act (PDSA) cycles were used through the project duration to help address obstacles and barriers and improve communication and access. The team used a process run chart to test consistency between team members in following the algorithm e.g. weight gain goal documentation by the dietitian and by the providers and communicating the information to patients and families.

\section{Project Timeline}

A retrospective review of our CFC registry reports (provided yearly by the CFF) from 2005 to 2008 was done with a focus on the BMI data for patients with CF between 3 and 19 years of age. The center data was compared to the median BMI for all CF Centers in the United States. A comparison was also made to the top 10 performing $\mathrm{CF}$ Centers nationwide. Although our overall BMI percentile was above the national median from 2005 to 2008, it was decided to undertake this project to in an effort to improve the lowest two BMI groups followed by our center. Data were collected quarterly from January 2010 through June 2012 for analysis. BMI groups were determined based on entry BMI percentile. All data analysis was performed based on group assignment on project entry 


\begin{tabular}{|c|c|c|}
\hline & Specific Interventions & Comment/Barrier/Success \\
\hline \multirow{2}{*}{$\begin{array}{l}\text { BMI } \geq \mathbf{5 0} \% \\
\text { Nutritionally Adequate }\end{array}$} & $\begin{array}{l}\text { Continue current high calorie diet and nutrition } \\
\text { supplement }\end{array}$ & \\
\hline & Goal weight & \\
\hline \multirow{3}{*}{$\begin{array}{l}\text { BMI 25-49\% } \\
\text { At Risk }\end{array}$} & High calorie diet & \\
\hline & Oral supplements & \\
\hline & Goal weight & \\
\hline \multirow{7}{*}{$\begin{array}{l}\text { BMI } 10-24 \% \\
\text { Urgent at Risk }\end{array}$} & High calorie diet & \\
\hline & Oral supplements & \\
\hline & Social work/Psychology involvement & \\
\hline & \begin{tabular}{|l|} 
Appetite stimulants \\
\end{tabular} & \\
\hline & \begin{tabular}{|l|} 
G-Tube discussion \\
\end{tabular} & \\
\hline & Close monitoring of caloric intake & \\
\hline & Goal weight & \\
\hline \multirow{8}{*}{$\begin{array}{l}\mathrm{BMI}<10 \% \\
\text { Critically at Risk }\end{array}$} & High calorie diet & \\
\hline & Oral supplements & \\
\hline & Social work/Psychology involvement & \\
\hline & Appetite stimulants & \\
\hline & G-Tube planning & \\
\hline & Close monitoring of caloric intake & \\
\hline & Increase visit frequency & \\
\hline & Goal weight & \\
\hline
\end{tabular}

Fig. 1. CF nutritional algorithm.

(i.e. group assignment was fixed for the duration of the project).

\section{Statistical Analysis}

BMI percentile is expressed as percentile based on CDC pediatric growth charts. Spirometric measures of lung function are reported as percent predicted values (NHANESIII). Differences in distribution of baseline characteristics by BMI group were calculated using the Wilcoxon rank sum test, chi-square test or Fisher's exact test based on the distribution of the variable. Mixed effects models were used to model the association between BMI percentile and $\mathrm{FEV}_{1}$ \%levels over time controlling for initial BMI percentile group, age, gender, genetic group, and whether one had a g-tube or not. Time, age, and gender were always kept in the models due to possible confounding, but genetic group and having a gtube only remained in the model if $P<0.2$.

To investigate the effect of our intervention, we modeled the pattern of BMI percentiles over time according to initial BMI percentile group with a mixed model. This model accounted for random effects of time and a random intercept (starting BMI percentile), as well as the fixed effects of gender and age. Since the visits were not equally spaced for all patients (approximately quarterly), time entered the equation as a continuous fixed variable as months from baseline, as well as a random effect. The interaction between months from baseline and BMI group was included to assess if there were different changes in BMI percentiles over time for each BMI group.

Additionally, we were interested in the association of our intervention, BMI percentiles, and lung function. To analyze this, we ran a mixed model on the lung function outcome to see the association with time (months from baseline, entered with linear and quadratic terms) and BMI percentile (as it changed over time for all individuals) controlling for age and gender. We allowed for a random intercept and slope of the linear time variable. $\mathrm{FEF}_{25-75} \%$ and $\mathrm{FEV}_{1} \%$ had strong positive Pearson correlation ranging from 0.75 to 0.82 over the entire time period $(P<0.0001$ for each time point). Due to this high correlation between $\mathrm{FEV}_{1} \%$ and $\mathrm{FEF}_{25-75} \%$ we presented results only for the model with $\mathrm{FEV}_{1} \%$ and found similar results (not shown) for $\mathrm{FEF}_{25-75} \%$.

All analyses were done in SAS v9.3. The Institutional Review Board waived oversight for the quality improvement project and analysis. 


\section{RESULTS}

173 children ages 3-19 years were seen in our CF center during the enrollment period of the project, January through June, 2010, and were therefore included.

Patient demographics are listed in Table 1 for the overall population, as well as the four BMI categories. The median age of this sample was 11.4 years with about equal numbers of males and females. There were no differences in age, sweat test value, or gender. There was a statistical difference in genetic category between the groups such that those in the $25-49 \%$ range are more likely to be homozygous dF508. However, if BMI groups are split by less than 25th percentile versus greater than or equal to the 25th percentile, there is no significant difference in genetic group distribution by BMI percentile group, $P=0.63$ [Data not shown], so this may be a spurious finding.

Table 2 summarizes the results from a mixed model investigating the pattern of BMI percentiles over time for patients by initial BMI group. After accounting for age and gender, the interaction between months from baseline and initial BMI group was significant, $P=0.019$, On average, for those who began with a BMI percentile less than 10, BMI percentile increased by 0.29 every month $(P=0.033)$, for those who began with BMI in the 10th24th percentile, BMI percentile increased each month by $0.26(P=0.034)$, and for those who began with BMI percentile between 25 and 49, BMI percentile increased by $0.18(P=0.031)$. Therefore, there was a slight increase in BMI in all groups with less than 50th BMI percentile and slight, but not significant, decrease in BMI percentile for those with greater than 50th percentile (decrease of 0.13 BMI percentile for each month, $P=0.10$ ). This can be seen in Figure 2 which presents the mean BMI percentiles over time by initial BMI percentile groups. There is not large movement in the average BMI percentile for groups who started with greater than 25 th BMI percentile, but there is increase over time in those who began with lower BMI percentiles.
Since we targeted those who began with BMI less than the 25 th percentile, we show the proportion of patients with this low of BMI throughout the duration of the study in Figure 3. We see that these low BMI patients make up almost a quarter of our sample at the beginning, but this generally decreases over time to less than $20 \%$. We note that of those who began with BMI percentile less than 10,7 (41\%) finished the study with BMI percentile between 10 and 53. Of those who began in the 10-24 percentile, 15 (75\%) maintained or gained BMI percentile ranging from 10 to 69 . Of those who started in the 25-49th percentile, 32 (86\%) finished the study in the 25-50th percentile. Finally, $82(83 \%)$ of those who began with BMI percentile greater than 50, ended the study with BMI percentile from 50 to 71.

Median $\mathrm{FEV}_{1 \%}$ levels were higher for children with higher BMI percentile levels (Fig. 4) over time. For those who began with BMI less than the 25th percentile, on average $\mathrm{FEV}_{1 \%}$ values were around or less than $80 \%$. In contrast, for those who began with BMI at the $25^{\text {th }}$ percentile or greater, median $\mathrm{FEV}_{1 \%}$ ranged from 90 to $100 \%$. In the model, there was a significant decrease in $\mathrm{FEV}_{1 \%}$ in the first month of $0.41(P=0.0001)$ with less of a decrease as time went on (decrease was 0.20 at one year and a slight increase of 0.03 at the end of two years, $P=0.012$ ) controlling for BMI percentile, age and gender. Thus, holding gender and age constant, FEV generally decreased during the course of the study, but by less than 5 points for all groups. BMI percentile and age were independently significantly associated with $\mathrm{FEV}_{1 \%}$ levels over time. Specifically, increasing BMI percentile was associated with increasing lung function such that for each increase in BMI percentile, $\mathrm{FEV}_{1 \%}$ increased by $0.18 \quad(P<0.0001)$. In contrast, increasing age was associated with decreased lung function such that each additional year older was associated with a decrease of 0.95 in lung function $(P=0.001)$.

\section{DISCUSSION}

In our QI project we found that improving BMI for our patients with CF who began with BMI less than the 25th

TABLE 2-Results from Mixed Model of BMI Percentile by Initial BMI Percentile Group

\begin{tabular}{lccc}
\hline Variable & Estimate & Standard error & $P$-value \\
\hline Intercept & 76.92 & 2.98 & 0.08 \\
Months from baseline & -0.13 & & \\
BMI Group (vs. $\geq 50 \%$ ile) & & 3.82 & \\
$\quad<10 \%$ ile & -67.93 & 3.55 & \\
10-24\%ile & -51.81 & 2.80 & \\
25-49\%ile & -32.75 & 0.0001 \\
Months* BMI Group & & 0.20 & 0.019 \\
$\quad$ Months*<10\%ile & 0.42 & 0.14 & 0.033 \\
Months*<10-24\%ile & 0.39 & 1.93 & 0.034 \\
$\quad$ Months* 25-49\%ile & 0.31 & 0.22 & 0.44 \\
Male (vs. Female) & -1.50 & -0.11 & 0.63 \\
Age (in years) & & & \\
\hline
\end{tabular}




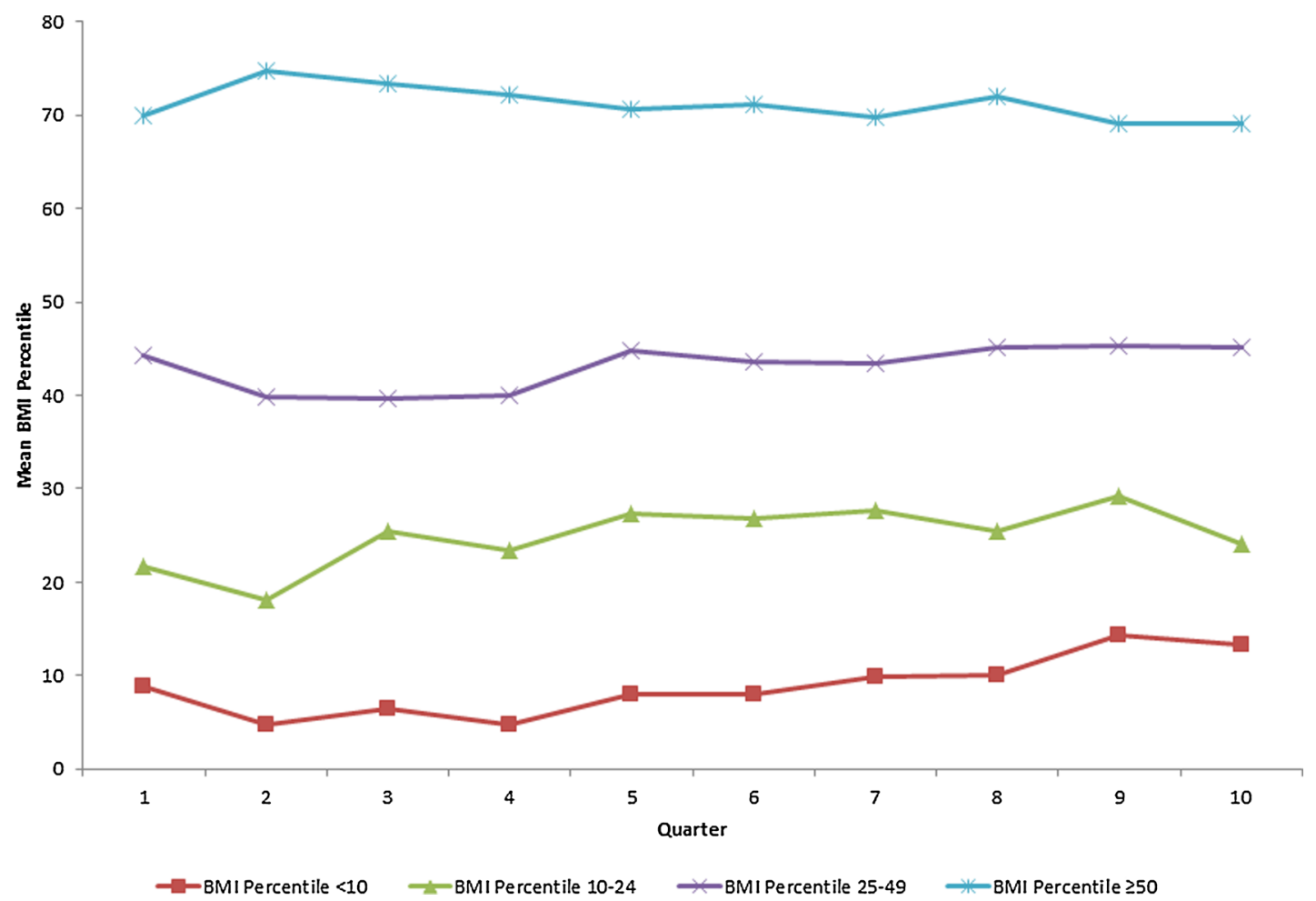

Fig. 2. Mean BMI percentiles for each initial BMI percentile group over study period.

percentile was achievable. This was possible by using several strategies. An important strategy was to standardize the CF center faculty and staff in following one algorithm in addition to the rest of the QI project. Another strategy was to include the CF FAB in developing the intervention steps and algorithm. In addition, buy in from patients and families was crucial to the success of the project. Throughout the QI project duration, team members were regularly updating patients and families with progress. The medical team was updated periodically about the overall progress through monthly meetings.

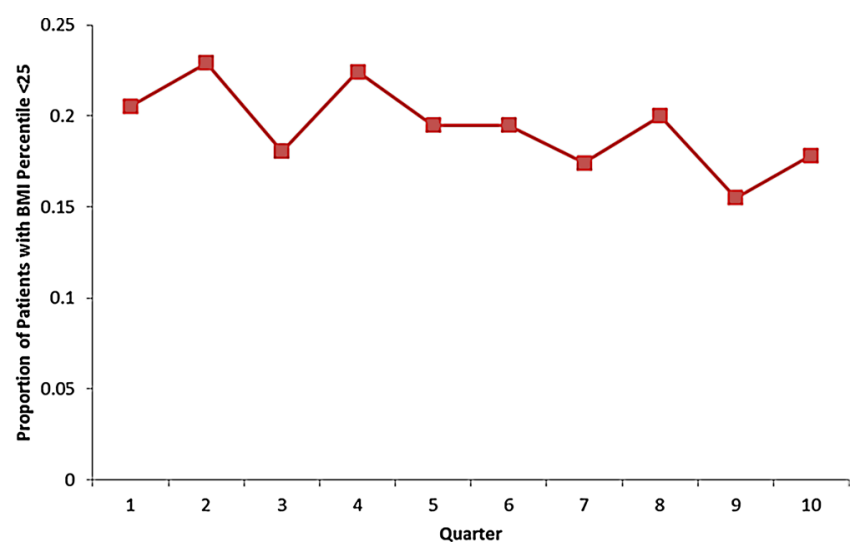

Fig. 3. Proportion of patients with BMI $<25^{\text {th }}$ percentile over study period.
Sharing problems and/or success stories occurred during these meetings as well. Implementation of the algorithm required collaboration between all team members, FAB, patients and families. Empowering not only our staff, but also patients and families to follow the algorithm through persistence and consistency was key in the success of this project.

We found a significant improvement of BMI percentile for those who began with BMI less than 10, those with BMI in the 10th-24th percentile and those with BMI in the 25 th-49th percentile over the project duration. This improvement was independent of age and gender and both of these characteristics were not significantly associated with a change in BMI percentile. This improvement was the result of the dedication of all that were involved buy in from patients and families, and multi-factorial approach.

When compared to other studies that aimed to improve nutrition and/or BMI for their patients, our QI project was distinct with respect to a couple of factors. First, the type of intervention in some studies has focused on using behavior modification as a means to improve nutrition. ${ }^{4,10,11}$ These studies included children 12 years of age and younger. Our project employed a multi-factorial approach to improve BMI. One of these strategies was an intervention by our psychologists and social workers to help address any issues related to the demand of the need to increase calorie intake. Counseling was done throughout the project duration. We chose to focus on children 


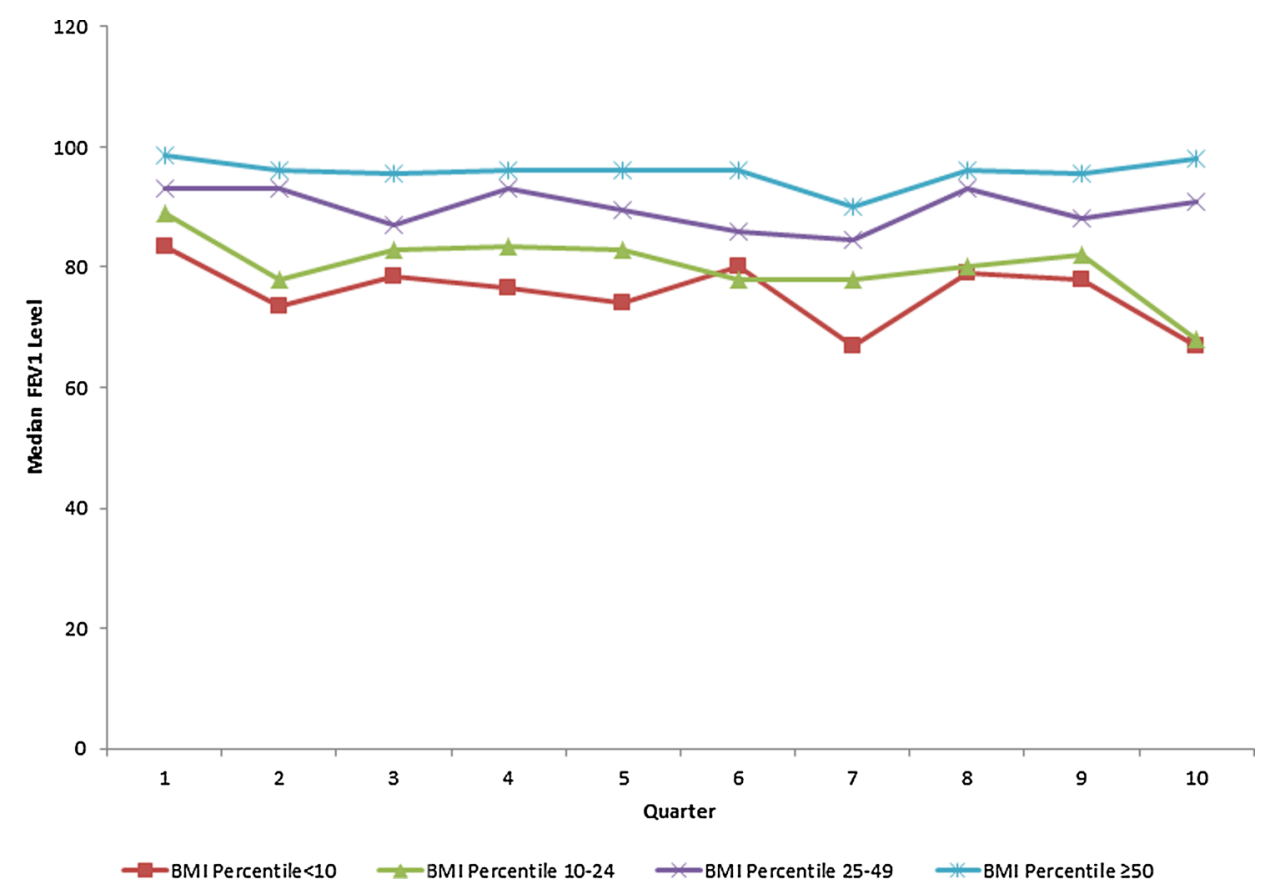

Fig. 4. Median FEV1 levels for initial BMI percentile groups over study period.

and adolescents between the ages of 3 and 19 years. The median age of children in the targeted lower two BMI groups, BMI 10-24\% and $<10 \%$, was 12.79 and 13.10 years respectively. Overall, the median age was 11.43 years for all participants. Also, these studies had a limited number of subjects and were of relatively short duration while our project was long-term and included a large number of patients.

Several reviews and consensus reports were published to evaluate nutrition-related management of patients with $\mathrm{CF}^{5,13,14}$ They all emphasized the need for regular evaluation of patient's nutritional status, the need for a high-calorie diet and the relationship between good nutritional status and quality of life. A project was performed to improve adherence to pancreatic enzyme replacement therapy (PERT) in pancreatic insufficient preadolescent patients with CF. Educational and behavioral interventions resulted in increasing adherence to PERT and increasing caloric intake as well. ${ }^{15}$ Two studies reported adopting a standard approach to nutritional assessment and treatment. ${ }^{16,17}$ This led to significant improvement in nutritional outcomes of patients with CF. Our standardized approach in addition to regular evaluation (and additional evaluation as needed) to address the nutritional needs of the patients resulted in achieving our goal of improvement in median BMI in our CF Center similar to other studies

Lung function, although not a primary outcome of our project, was evaluated to determine if the gains in BMI percentile in the lower groups corresponded to improvement in lung function. Over the duration of the project,
$\mathrm{FEV}_{1 \%}$ and $\mathrm{FEF}_{25-75 \%}$ across all groups remained steady without significant improvement. The fact that we did not see clinically significant improvement in lung function is likely due to the relatively short duration of the project and the relatively small number of patients included. It is difficult to extrapolate whether a longer time period of monitoring would demonstrate a significant change.

\section{CONCLUSION}

Improving median BMI for a CF center is achievable in a well-structured QI project involving many players. The key to improving nutrition and BMI for children with CF is multifactorial. There must be a motivated, multidisciplinary team. Collaboration with patients and families is crucial for the team to gain their feedback and provide them with their expertise to make goals attainable and sustainable. A variety of interventions must be used to find the right combination for the younger and older population. These interventions could be less for those patients who are able to maintain their BMI percentile at 25 or higher, but are much more elaborate and aggressive when a person's BMI percentile is less than 25 . These interventions should always be tailored to the patient based on their need and motivation. We believe that a clinicbased algorithm such as ours can be successful in improving the BMI percentile of patients with CF.

\section{ACKNOWLEDGMENT}

No external funding was secured for this project. Internal Funding: The Charles Woodson Biostatistics 
Consultation Program in the Department of Pediatrics, University of Michigan.

\section{AUTHOR CONTRIBUTION}

Ixsy Ramírez helped to plan the QI project worked on the manuscript and presented preliminary results in poster format at the CF conference in 2012. Amy Filbrun helped to plan the QI project, analyzed the results, worked on the manuscript. Aws Hasan gathered data for analysis, worked on manuscript. Kelley M. Kidwell performed the analysis and helped with manuscript. Samya Z. Nasr conceptualized the QI project, oversaw and planned the project, worked on the manuscript.

\section{REFERENCES}

1. Cystic Fibrosis Foundation. Cystic Fibrosis Foundation Patient Registry: 2011 Annual Data Report. Bethesda, MD, Cystic Fibrosis Foundation. 2012.

2. Kerem E, Reisman J, Corey M, Canny GJ, Levison H. Prediction of mortality in patients with Cystic Fibrosis. NE J Med 1992; 326:1187-1191.

3. Milla CE. Nutrition and lung disease in Cystic Fibrosis. Clin Chest Med 2007;28:319-330.

4. Stark LJ, Opipari-Arrigan L, Quittner AL, Bean J, Powers SW. The effects of an intensive behavior and nutrition intervention compared to standard of care on weight outcome in CF. Pediatr Pulmonol 2011;46:31-35.

5. Stallings VA, Stark LJ, Robinson KA, Feranchak AP, Quinton H. Evidence-based practice recommendations for nutrition-related management of children and adults with Cystic Fibrosis and pancreatic insufficiency: Results of a systematic review. J Am Diet Assoc 2008;108:832-839.

6. Gaskin KJ, Exocrine pancreatic function. In: Walker WA,Goulet O,Kleinman RE,Sherman PM,Schneider BL,Sanderson IR. edi- tors . Pediatric gastrointestinal disease. Hamilton, ON, Canada: B. C Decker; 2004. pp 1607-1623.

7. Onenstein DM. Cystic Fibrosis. In: Rudolph CD, Rudolph AM, Hostetter MK, Lister G, Seigel NJ. editors . Rudolph's Pediatrics. New York: McGraw Hill; 2002. pp 1969-1980.

8. Nasr S, Drury Z. Appetite stimulants use in Cystic Fibrosis. Pediatr Pulmonol 2008;43:209-219.

9. White H, Pollard K, Etheringtion C, Clifton I, Morton AM, Owen D, Conway SP, Peckham DG. Nutritional decline in cystic fibrosis related diabetes: The effect of intensive nutritional intervention. Journal of Cystic Fibrosis 2009;8:179-185.

10. Opipari-Arrigan L, Powers Scott W, Quittner AL, Stark LJ. Mealtime problems predict outcome in clinical trial to improve nutrition in children with CF. Pediatr Pulmonol 2010; 45:78-82.

11. Powers SW, Jones JS, Ferguson KS, Piazza-Waggoner C, Daines $\mathrm{C}$, Acton JD. Randomized clinical trial of behavior and nutrition treatment to improve energy intake and growth in toddlers and preschoolers with Cystic Fibrosis. Pediatrics 2005;116:14421450.

12. Watson $\mathrm{H}$, Bilton $\mathrm{D}$, Truby $\mathrm{H}$. A randomized controlled trial of a new behavioral home-Based nutrition education program, "Eat Well with CF," in adults with Cystic Fibrosis. J Am Diet Assoc 2008;108:847-852.

13. Munck A. Nutritional considerations in patients with Cystic Fibrosis. Expert Rev Resp Med 2010;4:47-56.

14. Borowitz D, Baker RD, Stallings V. Consensus report on nutrition for pediatric patients with Cystic Fibrosis. J Pediatr Gastroenterol Nutr 2002;35:246-259.

15. Schall JI, Bentley T, Stallings V. Meal patterns, dietary fat intake and pancreatic enzyme use in preadolescent children with Cystic Fibrosis. J Pediatr Gastroenterol Nutr 2006;43:651-659.

16. Matel JL, Milla CE. Nutrition in Cystic Fibrosis. Semin Respir Crit Care Med 2009;30:579-586.

17. Leonard A, Davis E, Rosenstein BJ, Zeitlin PL, Paranjape SM, Peeler D, Maynard C, Mogayzel PJ. Description of a standardized nutrition classification plan and its relation to nutritional outcomes in children with Cystic Fibrosis. J Pediatr Psychol 2010;35:6-13. 